\title{
An exploratory, pragmatic, cluster randomised trial of practice nurse training in the use of asthma action plans
}

\author{
*Jennifer A Clelanda, Susan Hallb, David Pricec, Amanda J Lee ${ }^{d}$ \\ a Senior Clinical Lecturer, Department of General Practice and Primary Care, University of Aberdeen, Scotland, UK \\ ${ }^{b}$ Research Assistant, Department of General Practice and Primary Care, University of Aberdeen \\ c Professor of Respiratory Disease in Primary Care, Department of General Practice and Primary Care, University of Aberdeen, \\ ' Professor of Medical Statistics, University of Aberdeen
}

Received 15th February 2007; accepted 26th July 2007

\begin{abstract}
Introduction: To investigate the feasibility of improving asthma management - in particular, the implementation of individualised asthma action plans (AAPs) for poorly-controlled adult asthma patients - by providing training in asthma-focused clinical and communication skills for practice nurses who deliver asthma clinics.

Methods: A pragmatic, cluster randomised trial with an intervention (an interactive seminar) delivered at practice level ( $n=13$ practices; $6=$ intervention, $7=$ control). The impact of the intervention was assessed against patient outcomes: routinely available asthma outcome measures ( $\beta 2$-agonist prescription rate and number of oral steroid courses) for asthma patients identified as being poorly-controlled from practice records; and questionnaire data - Mini Asthma Quality of Life Questionnaire (AQLQ) and the Asthma Control Questionnaire (ACQ) - from a subset of consenting patients. Data was collected at baseline and at 6-month follow-up.

Analysis: Routine data was analysed for 629 patients. 236 (37\%) of these patients consented to provide questionnaire data at baseline, with $75 \%$ returning questionnaires at follow-up. After adjustment for baseline and practice, there was a significant difference at followup between intervention and control practices on the Mini AQLQ only $(p=0.03)$. Estimates for subsequent sample sizes to inform future trials of asthma training were identified.

Conclusion: Training designed to support practice nurses in implementing individualised AAPs impacted on one patient outcome only. This disappointing outcome may have been due to many different factors such as outcome measure limitations, data collection problems, and underestimating the complexity of supporting practice nurses in behaviour change.

(C) 2007 General Practice Airways Group. All rights reserved.

JA Cleland, et al. Prim Care Resp J 2007; 16(5): 311-318.

doi:10.3132/pcrj.2007.00063
\end{abstract}

Keyw ords asthma, training, practice nurses, asthma action plans

\section{See editorial by Walker on page 269}

\section{Introduction}

Whilst there is no clear evidence that asthma action plans (AAPs) used in isolation have positive benefits on asthma outcomes in the community, ${ }^{1}$ there is evidence $^{2}$ that participatory communication within asthma consultations is associated with closer alignment with current asthma guideline recommendations $s^{3,4}$ including the provision of a written AAP to patients with more severe symptoms. Thus, it may be that written AAPs, used in the context of a patientcentred consultation, may be associated with better asthma management in the many patients with high levels of symptoms. 5.6

Current guidelines recommend helping asthma patients control their symptoms with the use of AAPs, but AAP use remains sub-optimal in primary care. ${ }^{7.8}$ Reported barriers include lack of confidence in the use of AAPs, and healthcare professionals believing that AAPs should be used only with certain types of patients. ${ }^{9,10}$ Another possible reason for the

\footnotetext{
* Corresponding author: Foresterhill Health Centre, Westburn Road, Aberdeen, AB25 2AY, Scotland, UK.

Tel: +44 (0)1224 553969 Fax: +44 (0)1224 550683 E-mail: jen.cleland@abdn.ac.uk
} 
paucity of AAP use is that primary healthcare professionals are not convinced of the applicability of AAPs to their patients, ${ }^{11,12}$ since the recommendation of AAP effectiveness is based on the outcome of randomised controlled trials (RCTs) ${ }^{13}$ and not from "real-life" populations where most patients would not qualify for enrolment in a classic RCT. ${ }^{14}$ Moreover, the frequent clinical care and monitoring of patients in RCTs is not typical of 'real-world' clinical situations. ${ }^{15}$

We wished to help in "shaping the square peg of the evidence to fit the round hole of the patient's life"12 by running a workshop to increase awareness of, and confidence in the use of, asthma guideline recommendations, particularly the use of AAPs, within the primary care consultation. We sought to address the question: in real life primary care, does training, designed to support practice nurses in implementing individualised AAPs, have the same impact on patient outcomes as that seen in RCTs?

This was a feasibility study to explore the practicalities of carrying out a complex intervention ${ }^{16}$ for changing practice nurse asthma consultation behaviour in UK primary care. A feasibility study was appropriate because, firstly, to the best of our knowledge, although practice nurses deliver the majority of UK asthma care - particularly in terms of providing asthma reviews $^{17}$ - there is little evidence on whether training in asthma management may impact on practice nurse behaviour. ${ }^{18}$ Secondly, the evidence for training leading to nurse behaviour change in other chronic disease management areas is conflicting..$^{19-21}$ Thirdly, we needed to identify practical and methodological issues to inform the design and sample size, including an estimation of the intracluster correlation coefficient (ICC) ${ }^{22}$ for a large, cluster randomised trial of AAP implementation.

In this paper, we report the findings of our study and the lessons learned.

\section{Methods}

Our intention was to enhance practice nurse skills; therefore, in order to minimise contamination, the unit of randomisation was the general practice. ${ }^{23}$ The study took place between 2000 (hypothesis) through application for funding (2001) and obtaining ethics permission (2002), to completion of data collection in December 2003.

\section{Recruitment of practices}

The primary eligibility criteria were use of the GPASS (General Practice Administration System for Scotland) computer system, location (North-East of Scotland), and the practice nurse(s) consenting to training. Fifty-six practices, representing both urban and rural practices, were eligible. Of these, 13 (23\%; seven urban and six rural) expressed interest and were assigned by an independent statistician, blind to the identity of the practices, to intervention $(n=6)$ or control $(n=7)$, using random number tables (Figure 1).

All practice nurses (PNs) in the study ran asthma clinics where they actively reviewed patients with existing asthma, assessed asthma in new patients, addressed management issues (e.g., checking inhaler technique), and agreed therapeutic management plans with patients. All but one PN had developed expertise in asthma via further qualifications (e.g. the Education for Health [formerly the National Asthma Training Centre] Diploma in asthma management). The majority of proactive asthma care was delivered in nurse-led clinics. General practitioners (GPs) tended to see asthma patients who attended for unscheduled appointments, but this was not exclusive - these patients may also have been seen by the PN, who would recommend appropriate treatment.

\section{Recruitment of patients}

Adult patients between the ages of 18 and 55 years with asthma diagnosed more than 12 months previously, who were receiving regular preventative asthma therapy, were identified using the GPASS computer search algorithms. We audited practice records to identify patients with poor control, since poor control is associated with more frequent symptoms and bronchodilator use, and functional impairment, ${ }^{24,25}$ as well as poor quality of life. ${ }^{26}$ Thus, there is scope for improving asthma management in this group of patients. Patients categorised as being likely to have poorly-controlled asthma by high $\beta 2$-agonist prescription rates of more than one inhaler per month, and/or their number of oral steroid prescriptions for asthma in the previous 12 months, ${ }^{27}$ were deemed eligible for study inclusion.

\section{Intervention}

The intervention was a three-hour interactive seminar, using active learning techniques ${ }^{28}$ jointly delivered by a trainer with knowledge of asthma and consultation skills, and a respiratory nurse specialist, focusing on clinical and communication skills for asthma management ${ }^{29}$ It contained:

- clinical content (e.g., UK evidence-based guidelines)

- brief lectures: disease versus illness, evidence of effectiveness of communication

- examples of effective communication strategies

- case studies to practice formulation and review of individualised AAPs

- role play with actors, with feedback on use of AAPs and new communication strategies

- patient resources

The intervention was delivered following a standardised protocol to ensure consistency. Control practices continued with usual care and received training at the end of the study

\section{Outcomes}

Routinely available asthma data ( $\beta 2$-agonist prescription rates, number of oral steroid courses) was collected for all 
Figure 1. CONSORT flow chart.

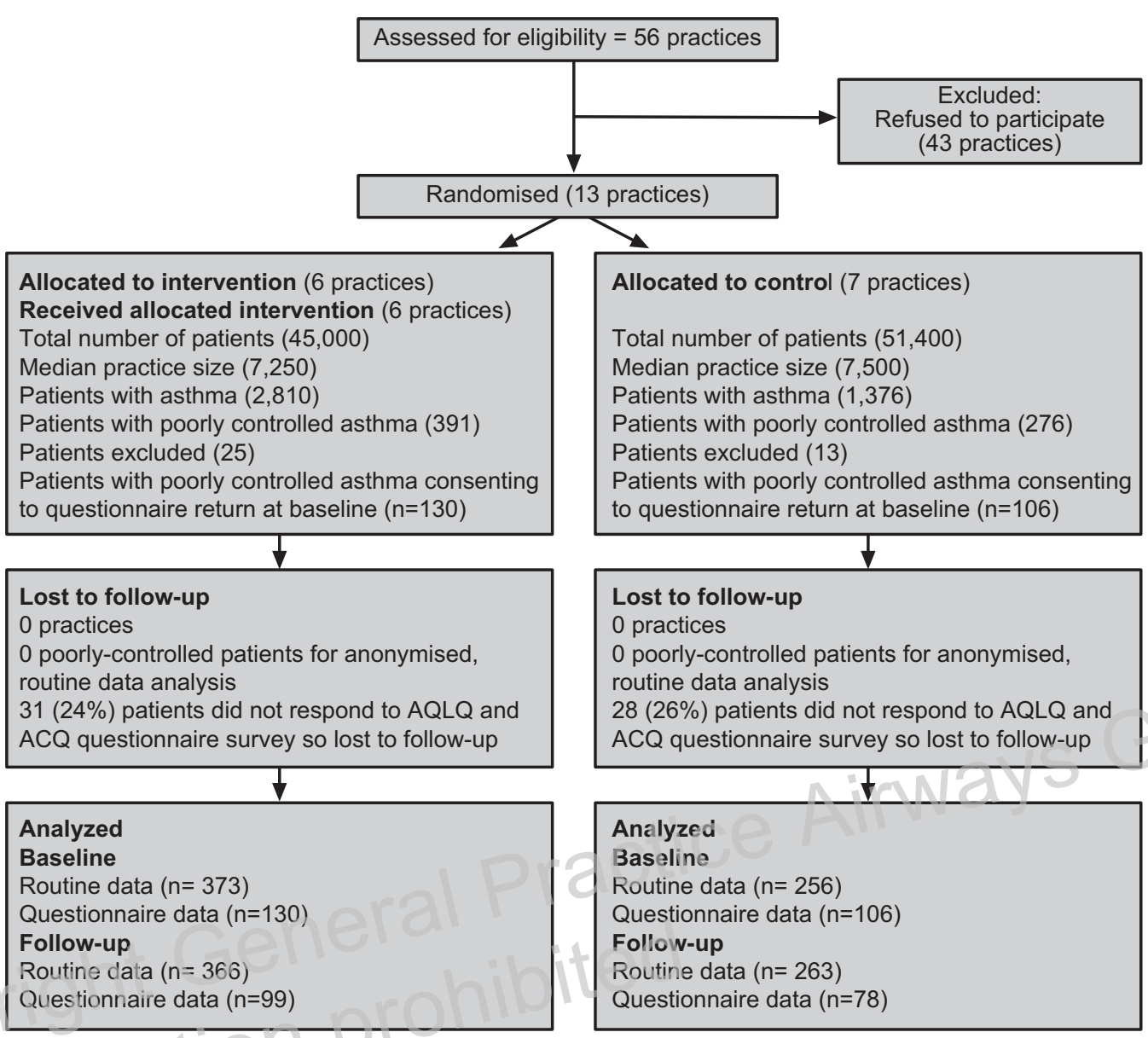

CONSORT flowchart adapted for cluster randomised trials. Campbell et al. BMJ 2004;328:702-08.

eligible patients. Patient questionnaire data was also collected from a subset of patients consenting to complete questionnaires. The primary outcome measure was the Juniper Mini Asthma Quality of Life Questionnaire (Mini AQLQ). ${ }^{30}$ The Asthma Control Questionnaire (ACQ) was a secondary patient-reported outcome measure. ${ }^{31}$ Patient outcome data was collected at baseline and six month followup. One reminder was sent, approximately three weeks after each mailing (baseline and follow-up). ${ }^{32}$

\section{Sample size and statistical power}

Since this was a feasibility study, no formal sample size calculation was performed; rather, sample size was based primarily on practicality and resource constraints.

Since patients were randomised to receive the intervention dependent on which practice they were in, rather than individually, the data were clustered by practice. Patients in a particular practice may be more similar to each other compared to patients in other general practices, for example, because some areas may be more deprived than others. Therefore, with a cluster design, there are two sources of variation in the outcome measures: variability from patients within a practice; and variability between practices. The effect of this increased variance is to increase the size of the standard errors of any outcome measure. The intra-practice correlation coefficient (ICC) is a statistical measure that is based on the relationship of the 'between' to the 'within' practice variation. ${ }^{22}$ Standard sample size formulae assume that the outcomes for each patient are independent and use of such formulae in cluster RCTs will result in underestimates of the sample size and subsequent under powered studies. Therefore, one of the objectives of this study was to produce a reliable estimate of the ICC to inform the sample size calculation of a definitive study.

\section{Statistical analysis}

Data from the audit of practice records and the questionnaires was coded and entered into Statistical Package for the Social Sciences (SPSS for Windows, Rel. 10. 2000. Chicago: SPSS Inc.). Means and standard deviations 
(for normally distributed data) and median and inter quartile ranges (IQR) for skewed data were reported. To check for potential bias, differences between subjects who consented to complete a questionnaire, and those who provided routine data only, were examined using the independent sample ttest, Mann-Whitney test or chi-squared test as appropriate. Similar univariate tests were used to examine differences in outcomes and routine data measures between intervention and control groups. These same measures at follow-up were compared between control and intervention groups using the independent sample t-test. The levels at follow-up were then adjusted for the confounding effects of baseline outcome level and practice using analysis of covariance.

\section{Results}

\section{Practices}

Six practices were classed as rural, seven as urban. Practice list size ranged from 2,300 to 12,500 (median=7,500, IQR $5,250-10,250)$. Four urban and three rural practices were randomly assigned to the intervention group.

\section{Patients}

Four thousand, four hundred and thirty-four adult (age range 18 to 55 years) patients with an asthma diagnosis made more than 12 months previously, from a summed practice size of just under 96,500, were identified from Read codes. A total of 1572 patients, who had received repeat prescriptions for $\beta 2$-agonists in the previous 12 months, were defined as active asthma patients. Of these, $667(42 \%)$ were considered to have poorly-controlled asthma using the criteria described earlier. Thirty-eight patients were excluded on the basis of life-threatening illness, co-morbid COPD, any other unresolved respiratory disorder, learning disability, or pregnancy. The remaining 629 patients with poor control were deemed the study group and were included anonymously in the study process. Routine asthma outcome data was collected from these 629 patients and they were also invited to take part in the postal questionnaire survey.

Three hundred and nineteen questionnaire responses were returned from the study group of 629 patients, an overall response of $51 \%$, similar to other primary care surveys of patients with respiratory disease..$^{33}$ Of these 319 responses, 236 (37\% of the patients with poorly-controlled asthma) patients consented to provide questionnaire data. One hundred and six (44.9\%) patients were from control practices, and 130 (55.1\%) were from intervention practices.

Table 1 presents characteristics of individuals consenting to complete questionnaires $(n=236)$ and those providing routine data only $(n=393)$. Patients with asthma who consented to provide baseline questionnaire data were significantly older, more likely to be female and more affluent than non-consenters. They had significantly fewer $\beta 2$-agonist inhalers or courses of oral steroids prescribed in the 12 months pre-study than non-consenters.

There were no significant differences between the intervention and control groups at baseline in terms of $\beta 2$ agonist use, courses of oral steroid prescribed, AQLQ and ACQ scores (Table 2).

One hundred and seventy-seven questionnaires were

Iable 1. Patient characteristics at baseline. Values are $n(\%)$ or median (IQR).

$\begin{array}{lcc}\text { Patient characteristics } & \text { Questionnaire responders } & \text { Routine data only } \\ n=236 & n=393 & p \text {-value }\end{array}$

\begin{tabular}{|c|c|c|c|c|}
\hline Age (years) & & $40(30-49)$ & $37(30-44)$ & 0.030 \\
\hline Gender & Male & $92(39.0)$ & $202(51.4)$ & 0.003 \\
\hline \multirow[t]{6}{*}{ Deprivation category } & 1 & $33(14.0)$ & $64(16.3)$ & \multirow{6}{*}{0.023} \\
\hline & 2 & $69(29.2)$ & $72(18.3)$ & \\
\hline & 4 & $48(20.3)$ & $85(21.6)$ & \\
\hline & 5 & $14(5.9)$ & $35(8.9)$ & \\
\hline & \multirow[t]{2}{*}{6} & $14(5.9)$ & $63(16.0)$ & \\
\hline & & 1 missing value & 5 missing values & \\
\hline \multicolumn{2}{|c|}{$\begin{array}{l}\text { Median number of b2-agonist inhalers } \\
\text { prescribed in } 12 \mathrm{~ms} \text { pre-baseline }\end{array}$} & $18(11-26)$ & $20(12-32)$ & 0.019 \\
\hline
\end{tabular}


Copyright GPIAG. Reproduction prohibited

An exploratory, pragmatic, cluster randomised trial of practice nurse training in the use of asthma action plans

\begin{tabular}{|c|c|c|c|}
\hline Patient outcome measures & Intervention & Control & $\mathrm{p}$-value \\
\hline Mini AQLQ score & $4.70(3.78-5.60)$ & $4.77(3.57-5.75)$ & 0.78 \\
\hline Asthma control questionnaire & $2.66(1.92-3.67)$ & $2.50(1.67-3.67)$ & 0.27 \\
\hline \multicolumn{4}{|l|}{ Routine data } \\
\hline $\begin{array}{l}\text { No. of } \beta 2 \text {-agonist inhalers prescribed in } \\
\text { previous } 12 \text { months }\end{array}$ & $19(12-32)$ & $18(13-30)$ & 0.51 \\
\hline $\begin{array}{l}\text { No. of patients receiving oral steroid } \\
\text { courses in previous } 12 \text { months }\end{array}$ & $0(0.00-2.00)$ & $0(0.00-1.75)$ & 0.62 \\
\hline
\end{tabular}

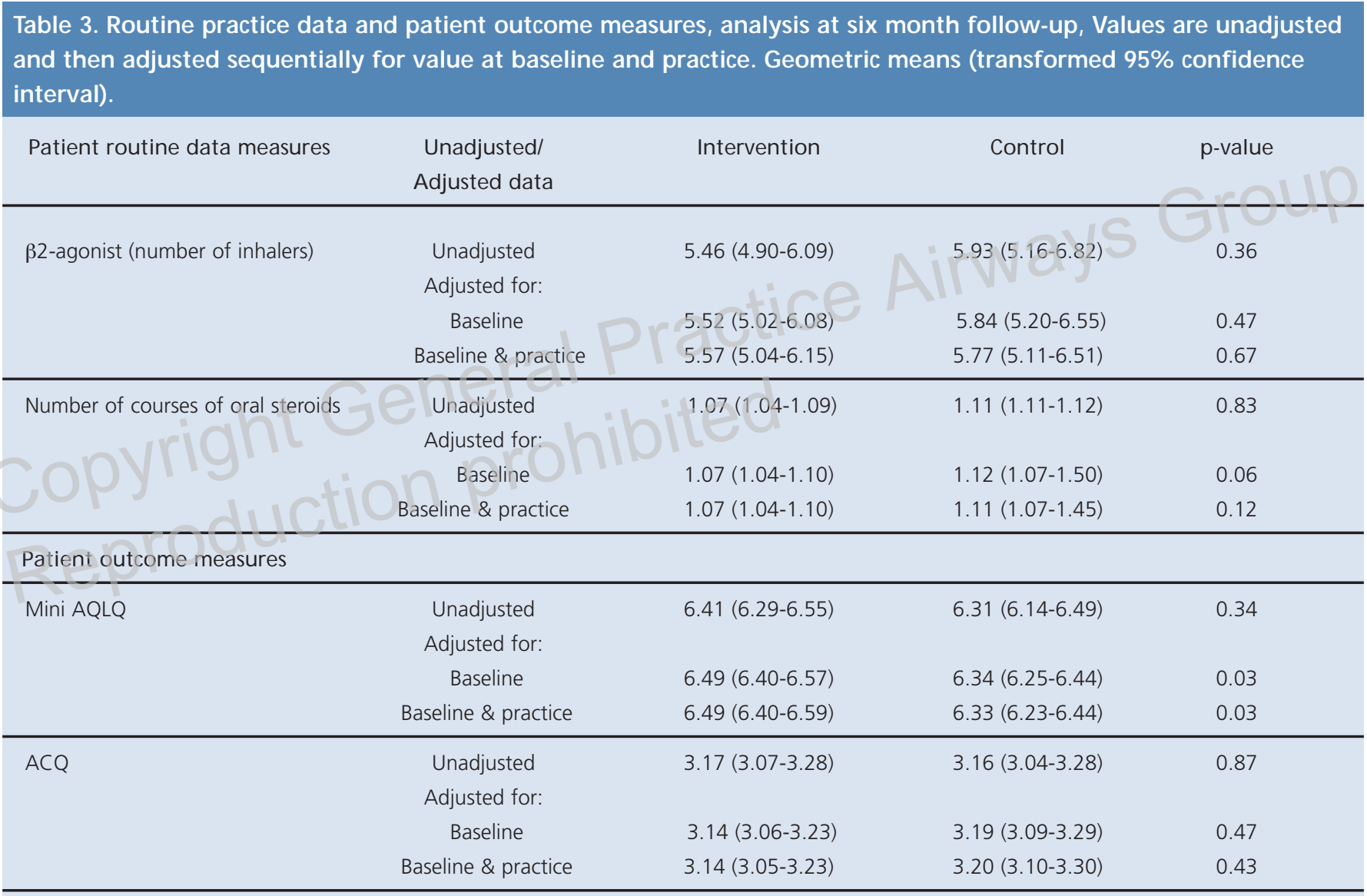

*t-tests were carried out on unadjusted means, univariate analysis of variance on adjusted means

returned at follow-up out of a possible 236 (75\%). Of these, $78 / 106$ (74\%) were returned by control practice patients and 99/130 (76\%) from intervention practice patients.

A significant difference was observed in the MiniAQLQ, but not $\beta 2$-agonist use or ACQ, between control and intervention practices at follow-up once data was adjusted for the value of baseline health measures or both baseline health measures and practice (Table 3). Groups differed significantly on numbers of courses of oral steroids prescribed but this difference disappeared when the data was adjusted for both baseline values and practice.

Table 4 shows the ICCs calculated for a range of outcome measures. Overall, the ICCS were generally quite low, indicating low variability between practices compared to the 


\begin{tabular}{ll}
$\begin{array}{l}\text { Table } 4 . \text { Intracluster correlation coefficient (ICC) } \\
\text { estimates for each outcome measure. }\end{array}$ & ICC \\
\hline Outcome measure & \\
\hline Mini asthma QOL score: & 0.026 \\
Total score & 0.054 \\
Symptoms & 0.024 \\
Environment & 0.032 \\
EmotionalActivity & 0.012 \\
& 0.062 \\
\hline Asthma control questionnaire & 0.024
\end{tabular}

variability between patients within a practice.

The estimated ICC for the overall MiniAQLQ was 0.03 with a standard deviation for change in overall MiniAQLQ of 0.7 .

\section{Discussion}

Training designed to support practice nurses in implementing individualised AAPs impacted on one patient outcome - the Mini AQLQ - only. This is of interest, since early studies comparing practices delivering nurse-led clinics versus those delivering usual care found no difference in quality of life (QoL) between groups of practices. ${ }^{34,35}$ No significant differences between control and intervention practices were seen in terms of asthma control, $\beta$-agonist or oral steroid use, once data was adjusted for baseline values and practice.

It may have been that additional outcome measures, such as healthcare utilisation ${ }^{35}$ could have been able to identify further differences between control and intervention practices. We wished to ensure that participation in the study was not time-consuming for PNs so did not ask PNs to collect data as part of the study. However, in retrospect, asking PNs to record which patients were given AAPs would have been informative.

We believe that, given the evidence from previous research ${ }^{2}$ and the timing (the introduction of new guidelines), our preliminary hypothesis was reasonable. The intervention was based on sound educational principles (e.g., active learning ${ }^{28}$ ). In addition, we undertook a feasibility study to identify any issues with practicalities and/or limitations of the methods proposed. However, to gain an accurate understanding of an intervention and its effects, its individual components need to be delineated, their interaction assessed, and this needs to be built into the study design. ${ }^{16,21,37}$ The limited impact of our intervention may have been due to: not knowing which aspects of the training intervention led to change, if any, and which did not; and/or the low number of patients enrolled in the study (see later); and/or the length of the intervention - which may have been too short to have an impact on behaviour. Furthermore, a one-off intervention, even if longer, may be insufficient in terms of reinforcing and reviewing behaviour change.

We may also have underestimated the complexity of supporting practice nurses in behaviour change and, in retrospect, might have gauged practice nurses' views of asthma guidelines and AAPs, rather than simply making assumptions. Their views could then have been sought as to their learning needs, how best to design, deliver and assess training, etc. Our outcome measures did not allow us to measure if the aim of improving practice nurse awareness and confidence with guidelines (and AAPs) was achieved by the intervention, even if this had little impact on patient outcomes. A theory of planned behaviour questionnaire ${ }^{38}$ may have been useful in this respect. ${ }^{39}$ Moreover, while acknowledging widespread changes in service delivery and nurse practitioner skills over the last 20 years, it may be that the outcomes of prescription rates examine GP behaviour and may not necessarily reflect nurse behaviour or practice.

Furthermore, we had overestimated the ease with which we could collect outcome data. We tried to collect this via routine practice data but found that identifying which patients had been given AAPs was not possible as it was not recorded in spite of suitable codes being available, nor were unscheduled and scheduled visits distinguishable. A useful lesson learned is that we should have included a simple question on whether or not the patient had been given an AAP in the questionnaire pack, at least then collecting that data for the patients who consenting to return questionnaire data. Thus, we were unable to ascertain whether or not practice nurses in the intervention arm of the study used these more than those in the control arm. In addition, routine data (e.g., asthma consultations) were frequently missing from GPASS, which limited data analysis. Thus, we do not know if our intervention was mostly unsuccessful because practice nurses did not introduce AAPs, or because patients did not follow them.

Using routine data did not allow us to compare usual care with the care received by patients seen in the intervention practices: there is no way of quantifying how these differed. Nor did routine practice data allow us to identify if the patients who took part in the study had all been seen by the nurses in the 6 month follow-up period, or how often they were seen.

Other issues also supported the importance of modelling before carrying out a feasibility study. Recruitment of practices via practice nurses was very poor until we discovered, via exploratory telephone calls, that nurses did not feel empowered to take part in this study without 
permission from their GPs, nor did they feel empowered to approach their GPs to seek support. We achieved our recruitment goal ( $n=12$ practices) by changing the protocol and writing to GPs inviting their practices to take part.

As well as lessons learnt, the study provided much useful data in terms of recruitment rates and estimates for subsequent sample size calculations to inform future primary care trials of professional training. Approximately one in four practices consented to take part, about one third of eligible patients from these practices consented to return questionnaire data, and about $75 \%$ of these consenting patients provided follow-up data at six months. This response loss is likely to be magnified at longer-term follow-ups. Regarding sample size, a total of 82 subjects are needed for an individually randomised trial to detect a clinically important difference of 0.5 in mini AQLQ (30) at $90 \%$ power and the $5 \%$ significance level. In contrast, $50 \%$ more subjects are needed (assuming 12 practices with 10 subjects per practice) for a cluster randomised trial with estimated mini AQLQ ICC of 0.03 . These numbers refer to number of participants needed to complete, indicating that, as expected, our feasibility study was underpowered for a cluster randomised trial using the mini AQLQ as a primary outcome measure. Our data also indicated that consenting asthma patients were different from non-consenters in terms of age, sex, deprivation and medication use, suggesting that the use of routine anonymous data is useful at least to check the applicability and generalisability of designed data sets."

Finally, training is only one way to change behaviour, and it is not always a successful method in routine health service practice. Other approaches, such as external review of electronic patient records coupled with specific feedback on individual patients who are poorly controlled, ${ }^{41}$ may be a more focused, and thus more acceptable, method of improving asthma management in primary care.

In conclusion, therefore, careful planning and staging of complex interventions is an essential stage in developing trials, especially those involving the complexities of human behaviour. We hope that the sample size data we identified, our description of our project, and how it might have been changed and improved, will help others as they design and undertake primary care asthma trials.

\section{Acknowledgements}

We wish to thank the staff and patients who took part in this study.

\section{Ethics and funding declaration}

Ethics approval for this study was granted by Grampian Research Ethics Committee. This study was partially funded by the Chief Scientist's Office, Scottish Executive, and Grampian Primary Care Trust Asthma Task Force.

\section{Conflict of interest declaration}

The authors declare no competing interests.

\section{References}

1. Toelle BG, Ram FS. Written individualized management plans for asthma in children and adults. Cochrane Database Syst Rev 2002;(3):CD002171.

2. Adams RJ, Appleton S, Wilson DH, Ruffin RE. Participatory decision making, asthma action plans, and use of asthma medication: a population survey. J Asthma 2005;42(8):673-8.

3. British Thoracic Society, Scottish Intercollegiate Guidelines Network. British guideline on the management of asthma. Thorax 2003;58(Supplement 1):i1 194.

4. National Institutes of Health/National Heart, Lung and Blood Institute. Global Initiative for Asthma: Pocket guide for asthma management and prevention. A pocket guide for physicians and nurses. 2002;NIH Publication No. 02-3659.

5. Rabe KF, Vermeire PA, Soriano JB, Maier WC. Clinical management of asthma in 1999: the Asthma Insights and Reality in Europe (AIRE) study. Euro Respir J 2000; 16:802-07.

6. Price $D$, Ryan D, Pearce L, Bride F. The AIR study: asthma in real life. Asthma J 1999;4:74-8.

7. Wilson DH, Adams RJ, Appleton S, et al. Prevalence of asthma and asthma action plans in South Australia population surveys from 1990-2001. Med J Aust 2003;178:483-5

8. Price $D$, Wolfe $S$. Delivery of asthma care: patients' use and views on healthcare services, as determined from a nationwide interview survey. J Asthma 2000:5:141-4

9. Moffat M, Cleland J, van der Molen T, Price D. Poor communication may impair optimal asthma care: a qualitative study. Fam Pract 2007;24(1):65-70. Epub 2006 Dec 7

10. Jones A, Pill R, Adams S. Qualitative study of views of health professionals and patients on guided self management plans for asthma. BMJ 2000;321:150710.

11. Cabana MD, Rand CS, Becher OJ, Rubin HR. Reasons for pediatrician nonadherence to asthma guidelines. Arch Pediatr Adolesc Med 2001; 155:1057-62

12. Freeman $A C$, Sweeney $K$. Why general practitioners do not implement evidence: qualitative study. BMJ 2001;323:1100-02

13. Gibson PG, Powell $H$. Written action plans for asthma: an evidence based review of the key components. Thorax 2004;59:94-9.

14. Herland K, Akselsen J, Skjonsberg OH, Bjermer L. How representative are clinical study patients with asthma or COPD for a larger "real life" population of patients with obstructive lung disease? Respir Med 2005;99:11-19.

15. Price DB, Thomas M. Breaking new ground: challenging existing asthma guidelines. BMC Pulm Med 2006;6(Suppl 1):S6

16. Medical Research Council. A framework for development and evaluation of RCTs for complex interventions to improve health. 2000:http://www.mrc. ac.uk/pdf-mrc_cpr

17. Ram FSF, Jones A, Fay JK. Primary care based clinics for asthma (Cochrane Review). In: The Cochrane Library, Issue 2. 2004.

18. Lewin SA, Skea ZC, Entwhistle V, Zwarenstein M, Dick J. Interventions for providers to promote a patient-centred approach in clinical consultations (Cochrane Review). The Cochrane Library 2004(3).

19. Kruijver IPM, Kerkstra A, Francks AL, Bensing JM, van de Wiel HBM. Evaluation of communication training programs in nursing care: a review of the literature. Patient Educ Couns 2000;39:129-45

20. Lane C, Johnson S, Rollnick S, Edwards k, Lyons M. Consulting about lifestyle change: evaluation of a training course for specialist diabetes nurses. Practical Diabetes Int 2003;20(6):204-08

21. Kinmonth A, Woodcock A, Griffin S, Spiegal N, Campbell MJ. Randomised controlled trial of patient centred care of diabetes in general practice: impact on current wellbeing and future disease risk. BMJ 1998;317:1202-08.

22. Fleiss JL. Statistical methods for rates and proportions. 2nd ed. New York: John Wiley \& Son; 1981. 
23. Zelen M. A new design for randomized clinical trials. N Engl J Med 1979; 300:1242-5

24. Barnes PJ, Woolcock AJ. Difficult asthma. Eur Respir J 1998;12:1209-18.

25. Hoskins G, McCowan C, Neville RG, Thomas GE, Smith B, Silverman R. Risk factors and costs associated with an asthma attack. Thorax 2000;55:19-24.

26. Vollmer WM, Markson LE, O'Connor E, et al. Association of asthma control with health care utilization and quality of life. Am J Resp Crit Care Med 1999; 160:1647-52.

27. Griffiths C, Foster G, Barnes N, et al. Specialist nurse intervention to reduce unscheduled asthma care in a deprived multiethnic area: the east London randomised controlled trial for high risk asthma (ELECTRA). BMJ 2004; 328:144-52

28. Bonwell C, Eison J. Active Learning: Creating Excitement in the Classroom AEHE-ERIC Higher Education Report No.1. Washington, DC: Jossey-Bass 1991.

29. Clark NM, Gong M, Schork A, et al. Impact of education for physicians on patient outcomes. Pediatrics 1998;101:831-6.

30. Juniper EF, Guyatt GH, Cox FM, Ferrie PJ, King DR. Development and validation of the Mini Asthma Quality of Life Questionnaire. Eur Respir J 1999;14:32-8.

31. Juniper EF, O'Byrne PM, Guyatt GH, Ferrie PJ, King DR. Development and validation of a questionnaire to measure asthma control. Eur Respir J 1999; 14:902-07.

32. Elliot AM, Hannaford PC, Simpson JA, Warskyj M, Ferry S, Owen-Smith V. Should postal epidemiological studies stop doing third mailings? Eur J Gen Pract 2001;7:104-10

33. Hazell M, Franck T, Frank P. Health related quality of life in individuals with asthma-related symptoms. Respir Med 2003;97:1211-18.
34. Charlton I, Charlton G, Broomfield J, Mulle MA. Audit of the effect of a nurserun asthma clinic on workload and patient morbidity in a general practice. $\mathrm{Br}$ J Gen Pract 1991;41:227-31.

35. Pilatto LS, Smith BJ, Heard AR, Elroy HM, Weekley J, Bennet P. Trial of nurserun asthma clinics based in general practice versus usual medical care. Respirology 2004;9:356-62.

36. Rowlands G, Sims J, Kelly S. A lesson learnt: the importance of modelling in randomized controlled trials for complex interventions in primary care. Fam Practice 2005;22:132-9.

37. Conner M, Norman P. Predicting health behaviour. Buckingham: Open University Press; 1996.

38. Cleland JA, Francis J, Watson MC, InC J, Bond C. Using theory to improve communication: designing a communication skills training package for medicine counter assistants. Int J Pharmacy Pract 2007;15:79-81.

39. Williams JG, Cheung WY, Cohen DR, Hutchings HA, Longo MF, Russell IT. Can randomized trials rely on existing electronic data? A feasibility study to explore the value of routine data in health technology assessment. Health Technol Assess 2003;7(26):1-117.

40. Grimshaw JM, Russell IT. Achieving health gain through clinical guidelines II. Ensuring guidelines change practice. Quality Health Care 1994;3:45-52.

41. Horne R, Ryan D, Freeman D, Lee A. Large variations in asthma control between UK general practices participating in the asthma control, concordance and tolerance (ACCT) Initiative. Prim Care Resp J 2006;15(3):206. doi: 10.1016/j.pcrj.2006.04.167

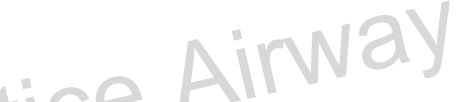

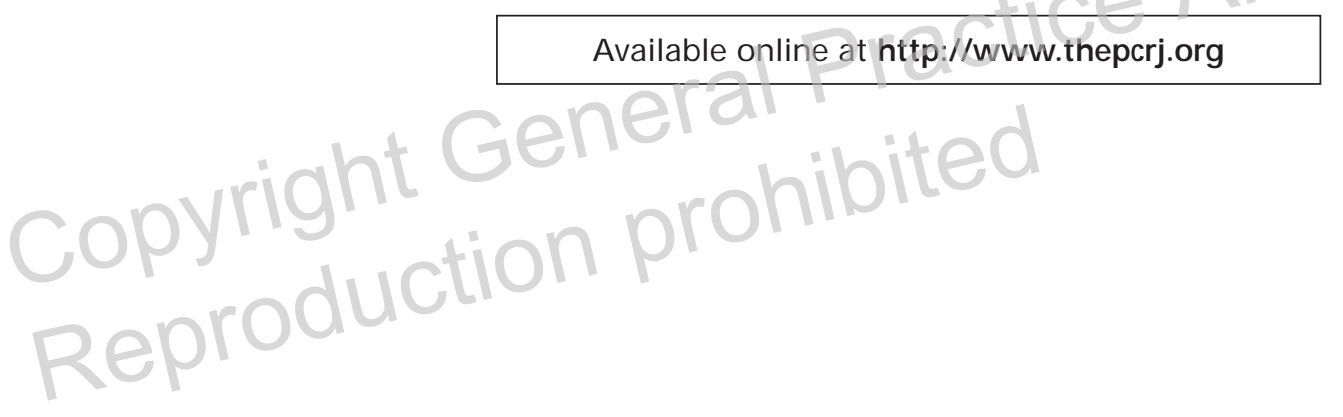

